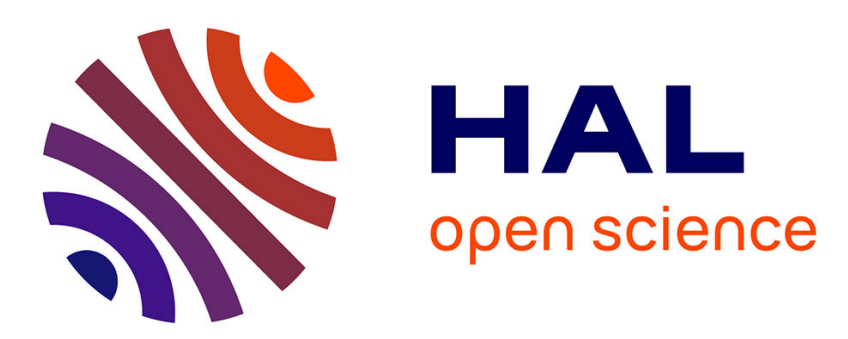

\title{
Perturbed optimization in Banach spaces II : a theory based on a strong directional constraint qualification
}

\author{
J. Frederic Bonnans, Roberto Cominetti
}

\section{To cite this version:}

J. Frederic Bonnans, Roberto Cominetti. Perturbed optimization in Banach spaces II : a theory based on a strong directional constraint qualification. [Research Report] RR-2161, INRIA. 1994. inria00074511

\section{HAL Id: inria-00074511 \\ https://hal.inria.fr/inria-00074511}

Submitted on 24 May 2006

HAL is a multi-disciplinary open access archive for the deposit and dissemination of scientific research documents, whether they are published or not. The documents may come from teaching and research institutions in France or abroad, or from public or private research centers.
L'archive ouverte pluridisciplinaire HAL, est destinée au dépôt et à la diffusion de documents scientifiques de niveau recherche, publiés ou non, émanant des établissements d'enseignement et de recherche français ou étrangers, des laboratoires publics ou privés. 


\title{
Perturbed Optimization in Banach Spaces II : A Theory Based on a Strong Directional Constraint Qualification
}

\author{
J. Frédéric BONNANS
}

Roberto COMINETTI

$\mathbf{N}^{\circ} 2161$

Janvier 1994

PROGRAMME 5

Traitement du signal, automatique et productique

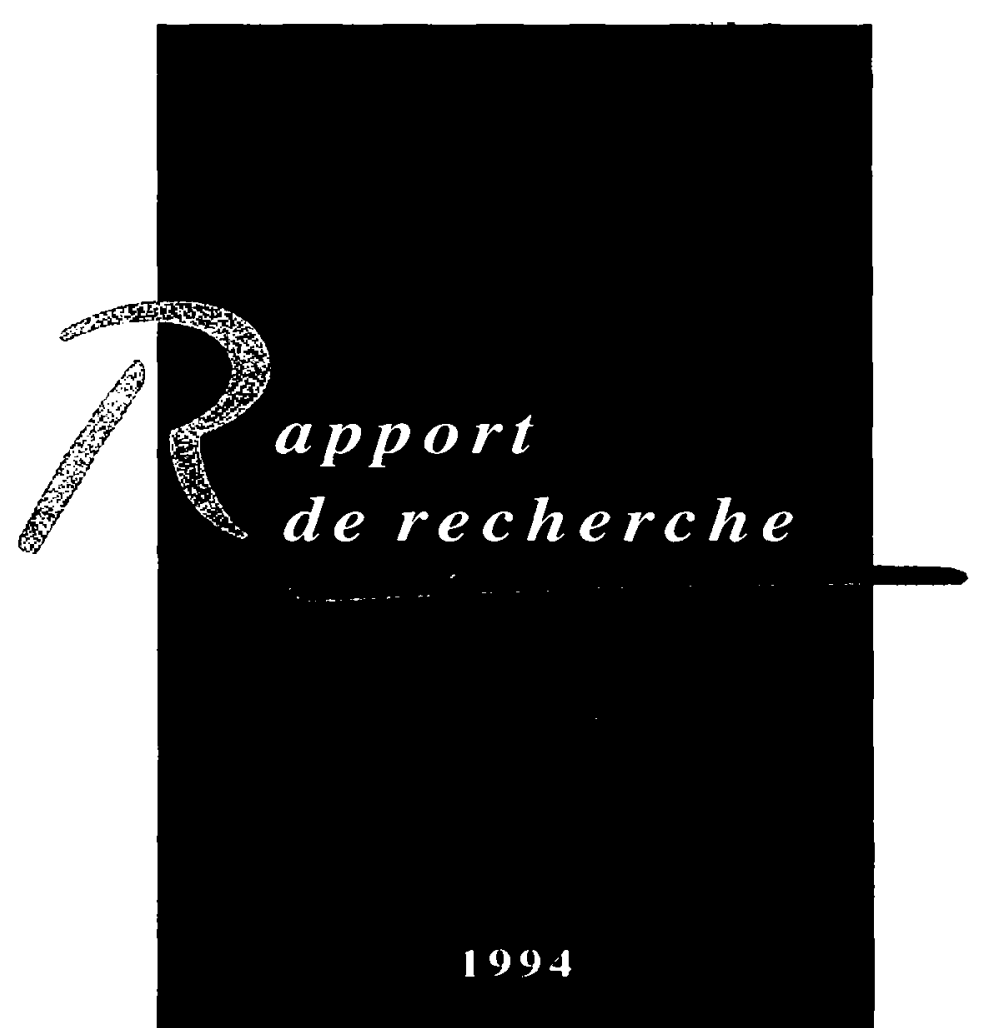




\title{
PERTURBED OPTIMIZATION IN BANACH SPACES II: \\ A THEORY BASED ON A STRONG DIRECTIONAL CONSTRAINT QUALIFICATION
}

\section{OPTIMISATION AVEC PERTURBATION DANS LES ESPACES DE BANACH II: UNE THÉORIE BASÉE SUR UNE CONDITION DE QUALIFICATION DIRECTIONNELLE FORTE}

\author{
J. FRÉDÉRIC BONNANS* AND ROBERTO COMINETTI'
}

\begin{abstract}
We study the sensitivity of the optimal value and optimal solutions of perturbed optimization problems in two cases. The first one is when multipliers exist but only the weak (and not the strong) second order sufficient optimality condition is satisfied. The second case is when no Lagrange multipliers exist. We introduce a directional constraint qualification stronger than in part I of this paper. We give sharp upper estimates of the cost based on paths varying as the square root of the perturbation parameter and, under a no gap condition, we obtain the first term of the expansion for the cost, and also for exact and approximate solutions when multipliers exist. We show in the appendix that the strong directional constraint qualification is satisfied for a large class of problems, including regular problems in the sense of Robinson.
\end{abstract}

Résumé. Nous étudions la sensibilité du coût optimal et des solutions de problèmes d'optimisation dans deux cas. Le premier est quand des multiplicateurs existent mais seule la condition suffisante d'optimalité faible est satisfaite. Le second cas est lorsque l'ensemble des multiplicateurs est vide. Nous introduisons une condition de qualification directionnelle plus forte que dans la première partie de l'article. Nous obtenons des estimations supérieures fortes pour le coût, basées sur des chemins variant comme la racine carrée du paramètre de perturbation et, sous une hypothèse d'écart nul, nous obtenons le premier terme du développement du coút, et aussi des solutions exactes et approchées quand l'ensemble des multiplicateurs n'est pas vide. Nous montrons en annexe que la condition de qualification directionnelle forte est satisfaite dans une classe de problèmes assez grande, qui contient les problèmes réguliers au sens de Robinson.

Key words. Sensitivity analysis, marginal function, square root expansion, approximate solutions, directional constraint qualification, regularity and implicit function theorems, convex duality.

A MS subject classifications. $46 \mathrm{~N} 10,47 \mathrm{H} 19,49 \mathrm{~K} 27,49 \mathrm{~K} 40,58 \mathrm{C} 15,90 \mathrm{C} 31$

1. Introduction. This paper is the second in a trilogy devoted to the analysis of parametric optimization problems of the form

$$
\min _{x}\{f(x, u): G(x, u) \in K\}
$$

with $X$ and $Y$ Banach spaces, $K$ a closed convex subset of $Y$, and $f(x, u), G(x, u)$ mappings of class $\mathcal{C}^{2}$ from $X \times \mathbb{R}$ into $\mathbb{R}$ and $Y$ respectively. We denote the feasible

- INRIA-Rocquencourt, B.P. 105, 78153 Rocequencourt, France

' Universidad de Cliile, Casilla 170/3 Correo 3, Santiago, Chile. Partially supported by Fundación Andes and Conicyt. 
set, value function, and set of solutions of $\left(P_{u}\right)$ as

$$
\begin{aligned}
F(u) & :=\{x \in X: G(x, u) \in K\}, \\
v(u) & :=\inf \{f(x, u): x \in F(u)\} \\
S(u) & :=\{x \in F(u): f(x, u)=v(u)\} .
\end{aligned}
$$

Similarly $v(P), F(P), S(P)$ will denote the optimal value, feasible set, and solution set of an optimization problem $(P)$.

Our aim is to study the expansion of $v(u)$ and possibly $S(u)$ in the vicinity of a local solution $x_{0}$ of $\left(P_{u}\right)$. Such sensitivity analysis usually rely (among other assumptions) upon stability properties of the feasible set $F(u)$ which follow from socalled constraint qualification conditions. In part I of this work [4] we considered the following generalization of Gollan's condition [10] (see also [1])

$$
(D C Q) \quad 0 \in \operatorname{int}\left[G\left(x_{0}, 0\right)+G^{\prime}\left(x_{0}, 0\right) X \times(0, \infty)-K\right],
$$

which is a directional version of Robinson's constraint qualification [14]

$$
\text { (CQ) } 0 \in \operatorname{int}\left[G\left(x_{0}, 0\right)+G_{x}^{\prime}\left(x_{0}, 0\right) X-K^{*}\right] .
$$

Under $(D C Q)$ we obtained the following upper estimate of the optimal value:

$$
v_{+}^{\prime}(0) \leq v(L)
$$

where $v_{+}^{\prime}(0)$ and $v_{-}^{\prime}(0)$ denote the upper and lower Dini derivatives of the value function:

$$
\begin{aligned}
v_{+}^{\prime}(0) & :=\limsup _{u \backslash 0} \frac{v(u)-v(0)}{u}, \\
v_{-}^{\prime}(0) & :=\liminf _{u \backslash 0} \frac{v(u)-v(0)}{u},
\end{aligned}
$$

and $(L)$ is the problem with linearized data:

$$
\min _{d}\left\{f^{\prime}\left(x_{0}, 0\right)(d, 1): G^{\prime}\left(x_{0}, 0\right)(d, 1) \in T_{K}\left(G\left(x_{0}, 0\right)\right)\right\} .
$$

Using duality theory we could prove that $v(D)=v(L)<\infty$, where $(D)$ is the problem

$$
\max \left\{\mathcal{L}_{u}^{\prime}\left(x_{0}, \lambda, 0\right): \lambda \in \Lambda_{0}\right\},
$$

with $\mathcal{L}$ the Lagrangian and $\Lambda_{0}$ the set of multipliers associated with $x_{0}$, that is, denoting by $N_{K}(y)$ the cone of outward normals at a point $y \in K$ :

$$
\begin{gathered}
\mathcal{L}(x, \lambda, u):=f(x, u)+\langle\lambda, G(x, u)\rangle, \\
\Lambda_{0}:=\left\{\lambda \in Y^{*}: \lambda \in N_{K}\left(G\left(x_{0}, 0\right)\right) ; \mathcal{L}_{x}^{\prime}\left(x_{0}, \lambda, 0\right)=0\right\} .
\end{gathered}
$$

It follows that under $(D C Q), v(L)$ is finite if and only if $\Lambda_{0}$ is not empty.

Define a path as a mapping $u-x_{u}$ from $\mathbb{R}_{+}$to $X$, with $x_{u} \rightarrow x_{0}$ when $u \downarrow 0$. The path is said to be feasible if $G\left(x_{u}, u\right) \in K$ for $u$ small enough. Under a strong second order condition on the Lagrangian it can be checked [4] that any $o(u)$-optimal path $x_{u}$, i.e. a feasible path $x_{u}$ such that $f\left(x_{u}, u\right) \leq v(u)+o(u)$, satisfies also $x_{u}=x_{0}+O(u)$. In this case $v^{\prime}(0)$ exists, being equal to $v(\bar{L})$, and some estimates for the second-order 
variation of $v(u)$ can be obtained. Under additional assumptions we could prove that in fact

$$
v(u)=v(0)+u v(L)+\frac{1}{2} u^{2} v(\tilde{Q})+o\left(u^{2}\right),
$$

where $(\tilde{Q})$ is a subproblem involving the expansion of order 1 and 2 of the data at $\left(x_{0}, 0\right)$. A remarkable property in this case is that every weak limit of $\left(x_{u}-x_{0}\right) / u$, with $x_{u}$ an $o\left(u^{2}\right)$-optimal path, belongs to $S(\tilde{Q})$.

The available perturbation theory for nonlinear programming shows that this is not the end of the story. Under the directional qualification hypothesis of Gollan [10] and the weak second order sufficient condition, it appears [9, Gauvin and Janin] that $v^{\prime}(0)$ exists but may be strictly less than $v(L)$. In that case, a path of $o(u)$-solutions satisfies only $x_{u}=x_{0}+O(\sqrt{u})$. One can still formulate [6, Bonnans, Ioffe and Shapiro] a subproblem $(M)$ such that $v^{\prime}(0)=v(M)$ and $S(M)$ coincides with the limit points of $\left(x_{u}-x_{0}\right) / \sqrt{u}$ where $x_{u}$ ranges over the set of all possible $o(u)$-optimal paths. For this it is necessary to assume the existence of at least one multiplier. A similar theory for the case when no multiplier exists has been developed in [3, Bonnans]: here the variation of the cost as well that of the solutions is of order $O(\sqrt{u})$.

The aim of this paper is to extend these two theories to the Banach space setting. To this end we need a constraint qualification that is still directional, but stronger than $(D C Q)$. Specifically, in addition to $(D C Q)$ we need the restorability property below that, roughly speaking, asserts that to certain almost feasible square root paths (i.e. paths satisfying $x_{u}=x_{0}+O(\sqrt{u})$ ), one can associate a sufficiently close feasible path. In the case of nonlinear programming, that stronger hypothesis $(S D C Q)$ below still reduces to the condition of Gollan $[10]$ used in $[9,3,6]$ so we recover the main results of these three references. Square root paths have already been used in a Banach space setting, see [2, Barbet] and loffe [11],[12]. However our qualification condition is weaker than those in the above references.

As in part I of this paper, in our extension to the Banach space setting, an additional difficulty related to the possible curvature of the convex $K$ appears. To be more precise, let us recall the definition of first and second order tangent sets:

$$
\begin{aligned}
T_{K}(y) & :=\{h \in Y: \text { there exists } o(t) \text { such that } y+t h+o(t) \in K\}, \\
T_{K}^{2}(y, h) & :=\left\{k \in Y: \text { there exists } o\left(t^{2}\right) \text { such that } y+t h+\frac{1}{2} t^{2} k+o\left(t^{2}\right) \in K\right\} .
\end{aligned}
$$

The fact that in general 0 does not belong to the set $T_{K}^{2}(y, h)$ may cause a gap between our upper and lower estimates. Some cases when the curvature makes no contribution to the second order variation of the cost were analyzed in part I, yielding the expansion (1.2) under a condition of generalized polyhedricity. We state in this paper some results of a similar nature. On the other hand, in part III [5] we will "fill the gap" for semi-infinite programming problems, a case where the curvature term may be handled (under some hypotheses) to yield sharper lower estimates.

The paper is organized as follows: in $\S 2$, we describe the strong directional constraint qualification $(S D C Q)$. Then in $\S 3$ we develop a perturbation theory assuming the set of multipliers $\Lambda_{0}$ to be nonempty, whereas $\S 4$ deals with the case when $\Lambda_{0}$ is empty. In both cases we obtain sharp upper estimates as well as some lower estimates of the cost. Under a no gap condition we obtain the first term in the expansion of the cost and approximate solutions. Finally in the appendix we give some sufficient conditions for $(S D C Q)$. 
2. The strong directional qualification condition. Our upper estimates are based on paths that vary as the square root of the perturbation parameter. Specifically, we consider paths satisfying, for given $d, w$ in $X$, the two conditions:

$$
\begin{gathered}
x_{u}=x_{0}+\sqrt{u} d+u w+o(u), \\
\operatorname{dist}\left(G\left(x_{u}, u\right), K\right)=o(u) .
\end{gathered}
$$

Note that we can express (2.4) using the concept of second order tangent set. Namely, if $x_{u}$ satisfies (2.3) then the expansion

$$
G\left(x_{u}, u\right)=G\left(x_{0}, 0\right)+\sqrt{u} G_{x}^{\prime}\left(x_{0}, 0\right) d+u\left[G^{\prime}\left(x_{0}, 0\right)(w, 1)+\frac{1}{2} G_{x}^{\prime \prime}\left(x_{0}, 0\right) d d\right]+o(u)
$$

shows that $(2.4)$ is equivalent to

$$
\Psi_{G}(w, d) \in T_{2}^{K}(d)
$$

where we have set

$$
\begin{aligned}
T_{2}^{K}(d) & :=\frac{1}{2} T_{K^{2}}^{2}\left(G\left(x_{0}, 0\right), G_{x}^{\prime}\left(x_{0}, 0\right) d\right) \\
\Psi_{G}(w, d) & :=G^{\prime}\left(x_{0}, 0\right)(w, 1)+\frac{1}{2} G_{x}^{\prime \prime}\left(x_{0}, 0\right) d d \\
\Psi_{f}(w, d) & :=f^{\prime}\left(x_{0}, 0\right)(w, 1)+\frac{1}{2} f_{x}^{\prime \prime}\left(x_{0}, 0\right) d d
\end{aligned}
$$

REMARK. The set $T_{2}^{K}(d)$ should not be confused with the set

$$
T_{K}^{2}(d):=T_{K}^{2}\left(G\left(x_{0}, 0\right), G^{\prime}\left(x_{0}, 0\right)(d, 1)\right)
$$

defined in part I of this paper and which will not be used here.

Definition 1. We say that $x_{0}$ is restorable (with respect to $G$ and $K$ ) if, given a path $x_{u}$ satisfying (2.3) and (2.4), then for each $\gamma \in(0,1)$ sufficiently close to 1 we can find $w_{\gamma} \in X$ and a feasible path

$$
x_{u}^{\gamma}=x_{0}+\gamma \sqrt{u} d+u w_{\gamma}+o(u)
$$

with $\lim _{\gamma \rightarrow 1} w_{\gamma}=w$.

We say that the strong directional constraint qualification (SDCQ) holds at $x_{0}$ if $x_{0}$ is restorable and the weak directional constraint qualification (DCQ) holds.

We discuss some sufficient conditions for $(S D C Q)$ in the appendix. In particular we show that for equality-inequality constrained problems (i.e. when $Y=Y_{1} \times Y_{2}$ with $Y_{1}$ and $Y_{2}$ Banach spaces and $K=\{0\} \times K_{2}$ with int $\left(K_{2}\right)$ nonempty) property $(S D C Q)$ is equivalent to $(D C Q)$. The sufficient condition in the appendix includes in fact a broader class of problems. For the sake of simplicity we prefer to postulate the restoration property.

Before proceeding with the sensitivity analysis we summarize in the next lemma four general properties $(P 1)-(P 4)$ which will be of constant use throughout the paper. Here $\sigma\left(\lambda, T_{2}^{K}(d)\right):=\sup \left\{(\lambda, k\rangle: k \in T_{2}^{K}(d)\right\}$ denotes the support function of $T_{2}^{K}(d)$.

Lemma 2.1. For every $d \in X$ we have 
(P1) $T_{2}^{K}(d)+T_{K}\left(G\left(x_{0}, 0\right)\right)-\mathbb{R}_{+} G_{x}^{\prime}\left(x_{0}, 0\right) d \subset T_{2}^{K^{\prime}}(d)$.

$(P 2)$ If $(D C Q)$ holds then $0 \in \operatorname{int}\left[T_{K}\left(G\left(x_{0}, 0\right)\right)-G^{\prime}\left(x_{0}, 0\right) X \times\{1\}\right]$.

(P3) $T_{2}^{K}(\gamma d)=\gamma^{2} T_{2}^{K^{\prime}}(d)$ for all $\gamma>0$.

(P4) If $T_{2}^{K^{\prime}}(d) \neq \phi$ then the following are equivalent

(a) $\sigma\left(\lambda, T_{2}^{K}(d)\right) \leq 0$.

(b) $\sigma\left(\lambda, T_{2}^{K}(d)\right)$ is finite.

(c) $\lambda \in N_{K}\left(G\left(x_{0}, 0\right)\right)$ and $\left\langle\lambda, G_{x}^{\prime}\left(x_{0}, 0\right) d\right\rangle=0$.

Proof. Properties $(P 1)$ and $(P 2)$ are straightforward consequences of [8, Prop. $3.1]$ and [4, Lemma B.3] respectively, while $(P 3)$ is an easy exercise.

Let us prove $(P 4)$. Since $T_{2}^{K}(d) \neq \phi$ the implication $(a) \Rightarrow(b)$ is straightforward. Also, the nonemptyness of $T_{2}^{K}(d)$ implies $G_{x}^{\prime}\left(x_{0}, 0\right) d \in T_{K}\left(G\left(x_{0}, 0\right)\right)$ and then $(b) \Rightarrow$ (c) follows from property $(P 1)$. To prove $(c) \Rightarrow(a)$ let us pick $y \in T_{2}^{K}(d)$ and choose $y_{t} \rightarrow y$ with $z_{t}:=G\left(x_{0}, 0\right)+t G_{x}^{\prime}\left(x_{0}, 0\right) d+t^{2} y_{t} \in K$. Using $(c)$ we deduce

$$
0 \geq\left\langle\lambda, z_{t}-G\left(x_{0}, 0\right)\right\rangle=\left\langle\lambda, t G_{x}^{\prime}\left(x_{0}, 0\right) d+t^{2} y_{t}\right\rangle=t^{2}\left\langle\lambda, y_{t}\right\rangle,
$$

so that $\langle\lambda, y\rangle=\lim \left\langle\lambda, y_{t}\right\rangle \leq 0$ proving $(a)$. $\square$

3. Perturbation analysis assuming the existence of multipliers. In this section we study the case when $\Lambda_{0} \neq \phi$. First we give an upper-estimate of $v_{+}^{\prime}(0)$, which we can express as a supremum of a certain function over $\Lambda_{0}$. We then rely on second-order conditions to obtain lower estimates for $v_{-}^{\prime}(0)$ and to investigate the coincidence of both estimates.

3.1. Sharp first-order upper estimates of the cost. Let $C_{0}$ denote the cone of critical directions at $x_{0}$, i.e.

$$
C_{0}:=\left\{d \in X: f_{x}^{\prime}\left(x_{0}, 0\right) d \leq 0 ; G_{x}^{\prime}\left(x_{0}, 0\right) d \in T_{K}\left(G\left(x_{0}, 0\right)\right)\right\} .
$$

When $\Lambda_{0} \neq \phi$ one has in fact $f_{x}^{\prime}\left(x_{0}, 0\right) d=0$ for all $d \in C_{0}$. To a path satisfying (2.3) and (2.4) is associated the constraint (2.5): whereas $\Psi_{f}(w, d)$ is the first term of the expansion of the cost. This leads to the problem

$$
\inf _{w \in X}\left\{\Psi_{f}(w, d): \Psi_{G}(w, d) \in T_{2}^{K}(d)\right\},
$$

and its dual:

$$
\sup _{\lambda \in \Lambda_{0}}\left\{\mathcal{L}_{u}^{\prime}\left(x_{0}, \lambda, 0\right)+\frac{1}{2} \mathcal{L}_{x}^{\prime \prime}\left(x_{0}, \lambda, 0\right) d d-\sigma\left(\lambda, T_{2}^{K}(d)\right)\right\}
$$

as well as

$$
\inf _{d}\left\{v\left(L^{d}\right): d \in C_{0}\right\}
$$

THEOREM 3.1. Assume $\Lambda_{0}$ to be nonempty and (SDCQ). Then

$$
v_{+}^{\prime}(0) \leq v(\tilde{L})=\inf _{d \in C_{0}} v\left(D^{d}\right) \leq v(L)<\infty .
$$

In parlicular. if $v(\dot{L})$ is finite, then

$$
v(u) \leq v(0)+u v(\tilde{L})+o(u) .
$$


The theorem is an immediate consequence of the next two lemmas. The first one gives the primal upper-estimate of $v_{+}^{\prime}(0)$.

Lemma 3.2. Assuming (SDCQ) we have

$$
v_{+}^{\prime}(0) \leq v(\tilde{L}) \leq v(L)<\infty .
$$

Proof. Let $d \in C_{0}$ and take a feasible $w \in F\left(L^{d}\right)$. Using the restorability property we may find $w_{\gamma} \rightarrow w$ and feasible paths of the form

$$
x_{u}^{\gamma}=x_{0}+\gamma \sqrt{u} d+u w_{\gamma}+o(u) .
$$

Expanding $f\left(x_{u}^{\gamma}, u\right)$ and using the fact that $d$ is critical, it follows

$$
v(u) \leq f\left(x_{u}^{\gamma}, u\right) \leq f\left(x_{0}, 0\right)+u \Psi_{f}\left(w_{\gamma}, \gamma d\right)+o(u)
$$

so that $v_{+}^{\prime}(0) \leq \Psi_{f}\left(w_{\gamma}, \gamma d\right)$. Passing to the limit when $\gamma \uparrow 1$ we deduce $v_{+}^{\prime}(0) \leq$ $\Psi_{f}(w, d)$, and taking the infimum over $w \in F\left(L^{d}\right)$ and $d \in C_{0}$ we get

$$
v_{+}^{\prime}(0) \leq v(\tilde{L})
$$

We conclude by noting that for $d=0$ problem $\left(L^{d}\right)$ reduces to problem $(L)$, and that $v(L)<\infty$ by [4, Prop. 2.2].

Let us prove next the dual expression for $v(\tilde{L})$.

Lemma 3.3. Assume $\Lambda_{0}$ to be nonempty and $(S D C Q)$. For each $d \in C_{0}$ we have (i) $v\left(D^{d}\right) \leq v\left(L^{d}\right)$.

(ii) if $\left(L^{d}\right)$ is feasible then, for all $\gamma \in(0,1), v\left(D^{\gamma d}\right)=v\left(L^{\gamma d}\right) \in \mathbb{R}$ and $S\left(D^{\gamma d}\right)$ is nonempty and bounded.

(iii) if $\left(L^{d}\right)$ is infeasible then $v\left(D^{\gamma d}\right)=\infty$ for all $\gamma>1$.

(iv) $\lim \sup _{\gamma \uparrow 1} v\left(D^{\gamma d}\right) \leq v\left(D^{d}\right)$.

As a consequence we obtain

$$
v(\tilde{L})=\inf _{d \in C_{0}} v\left(D^{d}\right)
$$

Proof. Let us begin by showing that $(3.7)$ is a consequence of $(i)-(i v)$. The inequality $v(\tilde{L}) \geq \inf _{d \in C_{0}} v\left(D^{d}\right)$ is obvious from $(i)$. To show the converse inequality it suffices to check that $v\left(D^{d}\right) \geq v(\tilde{L})$ for those $d \in C_{0}$ such that $v\left(D^{d}\right)<\infty$. By (iii) this implies $\left(L^{\gamma d}\right)$ is feasible for each $\gamma \in(0,1)$ and then (ii) gives $v\left(D^{\gamma d}\right)=v\left(L^{\gamma^{d}}\right) \geq$ $v(\tilde{L})$ for all $\gamma \in(0,1)$. We conclude by letting $\gamma \uparrow 1$ and using $(i v)$.

We now prove properties (i)-(iv).

(i) It suffices to show that if $w$ and $\lambda$ are feasible for $\left(L^{d}\right)$ and $\left(D^{d}\right)$ respectively, then the dual cost is not greater than the primal one. From the primal constraint it follows

$$
\sigma\left(\lambda, T_{2}^{K}(d)\right) \geq\left\langle\lambda, \Psi_{G}(w, d)\right\rangle,
$$

which implies

$$
\begin{aligned}
\Psi_{f}(w, d) & \geq \Psi_{f}(w, d)+\left\langle\lambda, \Psi_{G}(w, d)\right\rangle-\sigma\left(\lambda, T_{2}^{K}(d)\right) \\
& =\mathcal{L}_{u}^{\prime}\left(x_{0}, \lambda, 0\right)+\frac{1}{2} \mathcal{L}_{x}^{\prime \prime}\left(x_{0}, \lambda, 0\right) d d-\sigma\left(\lambda, T_{2}^{K}(d)\right)
\end{aligned}
$$


as was to be proved.

(ii) We first claim that $v\left(L^{d}\right)$ and $v\left(D^{d}\right)$ are finite and equal with $S\left(D^{d}\right)$ nonempty and bounded, whenever

$$
Y=\mathbb{R}_{+}\left[T_{2}^{K}(d)-G^{\prime}\left(x_{0}, 0\right) X \times\{1\}-\frac{1}{2} G_{x}^{\prime \prime}\left(x_{0}, 0\right) d d\right] .
$$

In order to motivate this relation, let us consider the family of problems obtained by perturbing additively the constraint of $\left(L^{d}\right)$, that is $\min _{w \in X} \varphi(w, y)$ with

$$
\varphi(w, y):=\left\{\begin{array}{cl}
\Psi_{f}(w, d) & \text { if } \Psi_{G}(w, d)+y \in T_{2}^{K}(d) \\
\infty & \text { otherwise. }
\end{array}\right.
$$

Property (3.8) amounts to $Y=\mathbb{R}_{+} \bigcup_{w} \operatorname{dom} \varphi(w, \cdot)$ so we may apply the convex duality theorem of part I [4, Thm. A.2] to deduce

$$
v\left(L^{d}\right)=\inf _{w \in \lambda^{\circ}} \varphi(w, 0)=-\min _{\lambda \in Y^{*}} \varphi^{*}(0, \lambda)
$$

as well as the boundedness and nonemptyness of the set of dual solutions. Now we compute

$$
\begin{aligned}
\varphi^{*}(0, \lambda) & =\sup _{w \in \lambda^{\prime}, y \in Y^{\prime}}\left\{\langle\lambda, y\rangle-\Psi_{j}(w, d): \Psi_{G}(w, d)+y \in T_{2}^{K}(d)\right\} \\
& =\sup _{w \in \lambda^{-}}\left\{\sigma\left(\lambda, T_{2}^{K}(d)\right)-\mathcal{L}^{\prime}\left(x_{0}, \lambda, 0\right)(w, 1)-\frac{1}{2} \mathcal{L}_{x}^{\prime \prime}\left(x_{0}, \lambda, 0\right) d d\right\} .
\end{aligned}
$$

Maximizing over $w$ we deduce that $\varphi^{*}(0, \lambda)=\infty$ if $\mathcal{L}_{x}^{\prime}\left(x_{0}, \lambda, 0\right) \neq 0$, and then using $(P 4)$ we get

$$
\varphi^{*}(0, \lambda)= \begin{cases}\sigma\left(\lambda, T_{2}^{K}(d)\right)-\mathcal{L}_{u}^{\prime}\left(x_{0}, \lambda, 0\right)-\frac{1}{2} \mathcal{L}_{x}^{\prime \prime}\left(x_{0}, \lambda, 0\right) d d & \text { if } \lambda \in \Lambda_{0} \\ \infty & \text { otherwise }\end{cases}
$$

This and (3.9) imply the equality $v\left(L^{d}\right)=v\left(D^{d}\right)$. Moreover, since the dual is attained, property $(P 4)$ shows that this common value is finite. This proves our claim.

In view of the previous discussion, to prove (ii) it suffices to check that for each $\gamma \in(0,1)$ property $(3.8)$ holds with $d$ replaced by $d_{\gamma}:=\gamma d$. To see this let us choose a feasible $w \in F\left(L^{d}\right)$, that is,

$$
G^{\prime}\left(x_{0}, 0\right)(w, 1)+\frac{1}{2} G_{x}^{\prime \prime}\left(x_{0}, 0\right) d d \in T_{2}^{K}(d) .
$$

Multiplying by $\gamma^{2}$ and using $(P 3)$ we deduce

$$
G^{\prime}\left(x_{0}, 0\right)\left(\gamma^{2} w, \gamma^{2}\right)+\frac{1}{2} G_{x}^{\prime \prime}\left(x_{0}, 0\right) d_{\gamma} d_{\gamma} \in T_{2}^{K}\left(d_{\gamma}\right)
$$

From this and $(P 1)$ we get

$T_{K}\left(G\left(x_{0}, 0\right)\right)-G^{\prime}\left(x_{0}, 0\right) X \times\left\{1-\gamma^{2}\right\} \subset T_{2}^{K}\left(d_{\gamma}\right)-G^{\prime}\left(x_{0}, 0\right) X \times\{1\}-\frac{1}{2} G_{x}^{\prime \prime}\left(x_{0}, 0\right) d_{\gamma} d_{\gamma}$, which multiplied by $\mathbb{I}_{+}$and using $(P 2)$ yields $(3.8)$ for $d_{\gamma}$ as required.

(iii) Let $\gamma>1$ and set $d_{\gamma}:=\gamma d$ as before. If $T_{2}^{K}(d)$ is empty, by $(P 3)$ so is $T_{2}^{K}\left(d_{\gamma}\right)$ and then $\sigma\left(\lambda, T_{2}^{K}\left(d_{\gamma}\right)\right)=-\infty$, hence $v\left(D^{\gamma d}\right)=\infty$. 
Let us then assume $T_{2}^{K}(d)$ to be nonempty. Since $\left(L^{d}\right)$ is infeasible, the convex set $T_{2}^{K}(d)-G^{\prime}\left(x_{0}, 0\right) X \times\{1\}$ does not contain $\frac{1}{2} G_{x}^{\prime \prime}\left(x_{0}, 0\right) d d$. But $(P 1)$ and $(P 2)$ show that this convex set has a nonempty interior, so that the Hahn-Banach theorem gives a nonzero $\mu \in Y^{*}$ that separates the set and the point, that is,

$$
\left\langle\mu, G^{\prime}\left(x_{0}, 0\right)(w, 1)+\frac{1}{2} G_{x}^{\prime \prime}\left(x_{0}, 0\right) d d\right\rangle \geq \sigma\left(\mu, T_{2}^{K}(d)\right) \text { for all } w \in X .
$$

This inequality and property $(P 4)$ imply $\mu \in N_{K}\left(G\left(x_{0}, 0\right)\right)$. Also, taking the infimum over $w \in X$ we deduce $\mu \circ G_{x}^{\prime}\left(x_{0}, 0\right)=0$ (that is to say, $\mu$ is a singular multiplier, as defined in the next section) so that for each $\lambda \in \Lambda_{0}$ and $t>0$ we have $\lambda+t \mu \in \Lambda_{0}$. Since $S(D)$ is bounded (see [4, Prop. 3.1]) it follows that

$$
\left\langle\mu, G_{u}^{\prime}\left(x_{0}, 0\right)\right\rangle<0 .
$$

With these observations property (3.10) reduces to

$$
\Xi(\mu, d):=\left\langle\mu, G_{u}^{\prime}\left(x_{0}, 0\right)+\frac{1}{2} G_{x}^{\prime \prime}\left(x_{0}, 0\right) d d\right\rangle-\sigma\left(\mu, T_{2}^{K}(d)\right) \geq 0,
$$

which multiplied by $\gamma^{2}$ and using $(P 3)$ gives

$$
\Xi\left(\mu, d_{\gamma}\right) \geq\left(1-\gamma^{2}\right)\left\langle\mu, G_{u}^{\prime}\left(x_{0}, 0\right)\right\rangle>0 .
$$

Let us fix $\lambda \in \Lambda_{0}$. Since $\Xi\left(\cdot, d_{\gamma}\right)$ is positively homogeneous and concave, and since $\lambda+t \mu \in \Lambda_{0}$, it follows that

$$
\begin{aligned}
v\left(D^{\gamma d}\right) & \geq f_{u}^{\prime}\left(x_{0}, 0\right)+\frac{1}{2} f_{x}^{\prime \prime}\left(x_{0}, 0\right) d_{\gamma} d_{\gamma}+\Xi\left(\lambda+t \mu, d_{\gamma}\right) \\
& \geq f_{u}^{\prime}\left(x_{0}, 0\right)+\frac{1}{2} f_{x}^{\prime \prime}\left(x_{0}, 0\right) d_{\gamma} d_{\gamma}+\Xi\left(\lambda, d_{\gamma}\right)+t \Xi\left(\mu, d_{\gamma}\right)
\end{aligned}
$$

To conclude we observe that $\left(P_{4}\right)$ implies the finiteness of $\Xi\left(\lambda, d_{\gamma}\right)$, so that letting $t \uparrow \infty$ and using (3.11) we get $v\left(D^{\gamma d}\right)=\infty$.

(iv) Using (P3) we obtain

$$
\begin{aligned}
v\left(D^{\gamma d}\right) & =\sup _{\lambda \in \Lambda_{0}}\left\{\mathcal{L}_{u}^{\prime}\left(x_{0}, \lambda, 0\right)+\frac{\gamma^{2}}{2} \mathcal{L}_{x}^{\prime \prime}\left(x_{0}, \lambda, 0\right) d d-\gamma^{2} \sigma\left(\lambda, T_{2}^{K}(d)\right)\right\} \\
& \leq \sup _{\lambda \in \Lambda_{0}}\left\{\left(1-\gamma^{2}\right) \mathcal{L}_{u}^{\prime}\left(x_{0}, \lambda, 0\right)+\gamma^{2} v\left(D^{d}\right)\right\} \\
& =\left(1-\gamma^{2}\right) v(L)+\gamma^{2} v\left(D^{d}\right)
\end{aligned}
$$

As $v(L)<\infty$, passing to the limit with $\gamma \uparrow 1$ we get the desired inequality. $\square$

Remark. If $(C Q)$ holds then $\left(L^{d}\right)$ is feasible for all $d \in C_{0}$, so that $v\left(D^{d}\right)=v\left(L^{d}\right)$. Otherwise the previous lemma shows that $v\left(D^{\gamma d}\right)=v\left(L^{\gamma d}\right)$ except for at most an exceptional value $\gamma_{0}$. The optimal values are finite for $\gamma<\gamma_{0}$ and equal to $+\infty$ for $\gamma>\gamma_{0}$. The following lemma shows that $\gamma_{0}=0$ iff $T_{2}^{K}(d)$ is empty. It will be useful in $\S 4$ as well.

LEMMA 3.4. Assume $(D C Q)$ and suppose $T_{2}^{K}(d)$ is not empty. Then, letting $d_{\gamma}:=\gamma d$ we have $F\left(L^{d_{r}}\right) \neq \phi$ for all $\gamma>0$ sufficiently small. 
Proof. Taking $k \in T_{2}^{K}(d)$ and using $(P 2)$ we get

$$
\frac{\gamma^{2}}{2} G_{x}^{\prime \prime}\left(x_{0}, 0\right) d d-\gamma^{2} k \in T_{K}\left(G\left(x_{0}, 0\right)\right)-G^{\prime}\left(x_{0}, 0\right) X \times\{1\}
$$

for all $\gamma>0$ sufficiently small. Then, using $(P 1)$ and $(P 3)$ we deduce

$$
\frac{1}{2} G_{x}^{\prime \prime}\left(x_{0}, 0\right) d_{\gamma} d_{\gamma} \in T_{2}^{K}\left(d_{\gamma}\right)-G^{\prime}\left(x_{0}, 0\right) X \times\{1\}
$$

so we may find $w \in X$ with $\Psi_{G}\left(w, d_{\gamma}\right) \in T_{2}^{K}\left(d_{\gamma}\right)$. $\square$

We end this section by giving a condition under which the upper estimate of Theorem 3.1 coincides with $v(L)$. Using (P4), it is easy to see that this condition is satisfied in particular if $\left(P_{0}\right)$ is convex in the sense that for all $y \in K$ and $\lambda \in N_{K}(y)$, the mapping $\mathcal{L}(\cdot, \lambda, 0)$ is convex. In that case the right-derivative $v^{\prime}(0)$ is actually equal to $v(L)$ (see [4, Prop. 3.2]).

Proposition 3.5. Assume $(S D C Q)$. Then $v(\tilde{L})=v(L)$ whenever

$$
\inf _{d \in C_{0}} \sup _{\lambda \in S(D)}\left\{\frac{1}{2} \mathcal{L}_{x}^{\prime \prime}\left(x_{0}, \lambda, 0\right) d d-\sigma\left(\lambda, T_{2}^{K}(d)\right)\right\} \geq 0 .
$$

Proof. By Lemma 3.3, and using the equality $v(L)=v(D)$ we get

$$
\begin{aligned}
v(\tilde{L}) & =\inf _{d \in C_{0}} v\left(D^{d}\right) \\
& \geq \inf _{d \in C_{0}} \sup _{\lambda \in S(D)}\left\{\mathcal{L}_{u}^{\prime}\left(x_{0}, \lambda, 0\right)+\frac{1}{2} \mathcal{L}_{x}^{\prime \prime}\left(x_{0}, \lambda, 0\right) d d-\sigma\left(\lambda, T_{2}^{K}(d)\right)\right\} \\
& \geq v(L)+\inf _{d \in C_{0}} \sup _{\lambda \in S(D)}\left\{\frac{1}{2} \mathcal{L}_{x}^{\prime \prime}\left(x_{0}, \lambda, 0\right) d d-\sigma\left(\lambda, T_{2}^{K}(d)\right)\right\} \\
& \geq v(L),
\end{aligned}
$$

and we conclude with Lemma 3.2.

3.2. Lower estimates and expansion of solutions. We derive next some lower estimates for $v_{-}^{\prime}(0)$. As $v_{-}^{\prime}(0) \leq v_{+}^{\prime}(0) \leq v(\tilde{L})$ whenever $(S D C Q)$ holds, this is only of interest if $v(\tilde{L})>-\infty$. We give conditions which imply $v_{-}^{\prime}(0)>-\infty$, based on a result of part I (Prop. 6.1) that we recall for the convenience of the reader.

For each set $\Omega \subset \Lambda_{0}$ we consider the second order condition

$\operatorname{SOC}(\Omega)$ There exist $\alpha, \epsilon>0$ s.t. $\max _{\lambda \in \Omega} \mathcal{L}_{x}^{\prime \prime}\left(x_{0}, \lambda, 0\right) d d \geq \alpha\|d\|^{2} \quad \forall d \in C_{\epsilon}$, where

$$
C_{\epsilon}:=\left\{d \in X: f_{x}^{\prime}\left(x_{0}, 0\right) d \leq \epsilon\|d\|, G_{x}^{\prime}\left(x_{0}, 0\right) d \in T_{K}\left(G\left(x_{0}, 0\right)\right)+\epsilon\|d\| B_{Y}\right\} .
$$

Note that for $\epsilon=0$ the extended critical cone $C_{\epsilon}$ reduces to the critical cone $C_{0}$.

Proposition 3.6. Assume (DCQ), and suppose $S O C(\Omega)$ holds for some bounded $\Omega \subset \Lambda_{0}$. Then, for each $O(u)$-optimal path $x_{u}$, we have $x_{u}=x_{0}+O(\sqrt{u})$.

Consider now the function

$$
\Pi(d):=\sup _{\lambda \in \Lambda_{0}}\left\{\mathcal{L}_{u}^{\prime}\left(x_{0}, \lambda, 0\right)+\frac{1}{2} \mathcal{L}_{x}^{\prime \prime}\left(x_{0}, \lambda, 0\right) d d\right\}
$$


and the subproblems

$$
\min \left\{\Pi(d): d \in C_{0}\right\}
$$

$$
\min \left\{\Pi(d): f_{x}^{\prime}\left(x_{0}, 0\right) d \leq \epsilon, G_{x}^{\prime}\left(x_{0}, 0\right) d \in T_{K}\left(G\left(x_{0}, 0\right)\right)\right\} .
$$

Note that $v\left(\tilde{D}_{\epsilon}\right)$ is a decreasing function of $\epsilon$; in particular $\lim _{\epsilon \downarrow 10} v\left(\tilde{D}_{\epsilon}\right) \leq v(\tilde{D})$. Moreover, from (P4) we get $\Pi(d) \leq v\left(D^{d}\right)$ and with Theorem 3.1 we deduce

$$
\lim _{\epsilon \downarrow 0} v\left(\tilde{D}_{\epsilon}\right) \leq v(\tilde{D}) \leq v(\tilde{L})
$$

Proposition 3.7. Assume (DCQ), the existence of an o(u)-optimal path, and $S O C(\Omega)$ for some bounded $\Omega \subset \Lambda_{0}$. Then $v_{-}^{\prime}(0)>-\infty$ and (i) If $(C Q)$ holds, then for each $\varepsilon>0$ we have

$$
v_{-}^{\prime}(0) \geq v\left(\check{D}_{\varepsilon}\right) .
$$

(ii) If any of the following conditions hold:

(a) the path may be expanded as $x_{u}=x_{0}+\sqrt{u} d_{0}+o(\sqrt{u})$,

(b) $X$ is reflexive and $d \rightarrow \mathcal{L}_{x}^{\prime \prime}\left(x_{0}, \lambda, 0\right) d d$ is weakly l.s.c. at each $d \in C_{0}$, then the previous lower bound may be strengthened to

$$
v_{-}^{\prime}(0) \geq v(\tilde{D}) .
$$

Proof. Let $x_{u}$ be an o(u)-optimal path. By Proposition $3.6 d_{u}:=\left(x_{u}-x_{0}\right) / \sqrt{u}$ stays bounded as $u \downarrow 0$ and then for each $\lambda \in \Lambda_{0}$ we have

$$
\begin{aligned}
v(u) & =f\left(x_{u}, u\right)+o(u), \\
& \geq v(0)+\mathcal{L}\left(x_{u}, \lambda, u\right)-\mathcal{L}\left(x_{0}, \lambda, 0\right), \\
& \geq v(0)+u\left[\mathcal{L}_{u}^{\prime}\left(x_{0}, \lambda, 0\right)+\frac{1}{2} \mathcal{L}_{x}^{\prime \prime}\left(x_{0}, \lambda, 0\right) d_{u} d_{u}\right]+o_{\lambda}(u),
\end{aligned}
$$

with $\left\|o_{\lambda}(u)\right\| / u \rightarrow 0$ uniformly when $\lambda$ varies over bounded sets. From this and the boundedness of $d_{u}$, it follows that $v_{-}^{\prime}(0)>-\infty$.

To prove (i) we apply Robinson's theorem [14] to the mapping $\tilde{G}(x):=G\left(x_{0}, 0\right)+$ $G_{x}^{\prime}\left(x_{0}, 0\right)\left(x-x_{0}\right)$ in order to find $\tilde{x}_{u}=x_{u}+o(\sqrt{u})$ such that $\tilde{G}\left(\tilde{x}_{u}\right) \in K$. Then, by suitably modifying the small term $o_{\lambda}(u)$, in (3.15) we can replace $d_{u}$ by $\tilde{d}_{u}:=$ $\left(\tilde{x}_{u}-x_{0}\right) / \sqrt{u}$. Moreover, under $(C Q)$ we know that $\Lambda_{0}$ is bounded so that taking supremum over $\lambda$ we get

$$
v(u) \geq v(0)+u \Pi\left(\tilde{d}_{u}\right)+o(u)
$$

from which (3.13) follows.

To show (ii), let us choose $u_{k} \downarrow 0$ realizing the lower limit $v_{-}^{\prime}(0)$. When $(a)$ holds we have $d_{u_{k}} \rightarrow d_{0}$, while in case $(b)$ we may assume that $d_{u_{k}}-d_{0}$. In both cases, $d_{0} \in C_{0}^{\prime}$ and using (3.15) we get

$$
v_{-}^{\prime}(0) \geq \mathcal{L}_{u}^{\prime}\left(x_{0}, \lambda, 0\right)+\frac{1}{2} \mathcal{L}_{x}^{\prime \prime}\left(x_{0}, \lambda, 0\right) d_{0} d_{0}
$$


where in case $(b)$ we use the weak 1.s.c. of $\mathcal{L}_{x}^{\prime \prime}\left(x_{0}, \lambda, 0\right) d d$. Taking the supremum over $\lambda \in \Lambda_{0}$ we conclude (3.14).

We now analyze under which conditions the gap between the estimate of Theorem 3.1 and (3.14) is null. We start with sufficient conditions for the equality between the optimal values of the subproblems giving the upper and lower estimates. We define extended polyhedricity of the second kind (for problem $\left(P_{0}\right)$, at point $\left.x_{0}\right)$ as $0 \in T_{2}^{K}(d)$ for all $d$ in a dense subset of $C_{0}$.

We note that in the definition of extended polyhedricity given in part I, the set $S(L)$ was considered instead of $C_{0}$. If the constraints are unperturbed, then $S(L)=C_{0}$ and both definitions coincide.

Proposition 3.8. Assume $\Lambda_{0}$ non empty and (SDCQ). If one of the two following conditions hold:

(a) $0 \in T_{2}^{K}(d)$ for all d in $C_{0}$,

(b) $(C Q)$ and extended polyhedricity hold, then $v(\tilde{L})=v(\tilde{D})$ and $S(\tilde{L}) \subset S(\tilde{D})$.

Proof. From (P4) it follows that when $0 \in T_{2}^{K}(d)$ we have $\sigma\left(\lambda, T_{2}^{K}(d)\right)=0$ for all $\lambda \in \Lambda_{0}$, and then $\Pi(d)=v\left(D^{d}\right)$. Consider now a minimizing sequence $\left\{d^{k}\right\}$ for $(\tilde{D})$ satisfying $\sigma\left(\lambda, T_{2}^{K}\left(d^{k}\right)\right)=0$. The existence of such a sequence is obvious in case $(a)$, while in case $(b)$ it is a consequence of the fact that, due to $(C Q), \Pi(d)$ is continuous. Along this sequence we have, by Theorem $3.1, \Pi\left(d^{k}\right)=v\left(D^{d^{k}}\right) \geq v(\tilde{L})$. It follows that $v(\tilde{L}) \leq v(\tilde{D})$. Reminding $(3.12)$, we get $v(\tilde{L})=v(\tilde{D})$. The inclusion $S(\tilde{L}) \subset S(\tilde{D})$ follows easily from this.

The final result of this section gives a formula for the marginal value $v^{\prime}(0)$, and analyzes the behaviour of paths of approximate solutions.

THEOREM 3.9. Assume $X$ reflexive, the existence of an o(u)-optimal path, $\mathcal{L}_{x}^{\prime \prime}\left(x_{0}, \lambda, 0\right) d d$ weakly l.s.c., and one of the two hypotheses below:

(i) $(C Q), S O C\left(\Lambda_{0}\right)$ and extended polyhedricity, Then

(ii) $(S D C Q), S O C(\Omega)$ for some bounded $\Omega \subset \Lambda_{0}$, and $0 \in T_{2}^{K}(d)$ for all $d$ in $C_{0}$.

(a) There exists $v^{\prime}(0)=v(\tilde{L})=v(\tilde{D})$, and $S(\tilde{L}) \subset S(\tilde{D})$.

(b) For every $o(u)$-optimal path $x_{u}$, the weak accumulation points of $\left(x_{u}-x_{0}\right) / \sqrt{u}$ belong to $S(\bar{D})$.

(c) If $d_{0} \in S(\tilde{L})$ and $w_{0} \in S\left(L^{d_{0}}\right)$, then there exists an o(u)-optimal path of the form $x_{u}=x_{0}+\sqrt{u} d_{0}+o(\sqrt{u})$.

Proof. (a) This follows combining Theorem 3.1 and Propositions 3.7 and 3.8 .

(b) Let $d_{0}$ be a weak limit point of $\left(x_{u}-x_{0}\right) / \sqrt{u}$. Expanding the Lagrangian as in (3.15) we get $v(\tilde{D})=v^{\prime}(0) \geq \Pi\left(d_{0}\right)$. As $d_{0}$ is feasible for $v(\tilde{D}), d_{0}$ is a solution of $v(\tilde{D})$.

(c) Using $(S D C Q)$ let us select $w_{\gamma} \rightarrow w_{0}$ and feasible paths of the form $x_{u}^{\gamma}=$ $x_{0}+\gamma \sqrt{u} d_{0}+u w_{\gamma}+o_{\gamma}(u)$, with (for each $\gamma$ ) $\left\|o_{\gamma}(u)\right\| / u \rightarrow 0$ when $u \rightarrow 0$. Take $\gamma_{k} \uparrow 1$ and choose a strictly decreasing sequence $u_{k} \downarrow 0$ such that

$$
\left\|o_{\gamma_{k}}(u)\right\| \leq \frac{u}{k} \quad \forall u \in\left[0, u_{k}\right]
$$

from which we construct the feasible path

$$
x_{u}=x_{u}^{\gamma_{k}} \quad \forall u \in\left[u_{k+1}, u_{k}\right)
$$


Then we have

$$
\left\|x_{u}-x_{0}-\sqrt{u} d_{0}\right\| \leq \sqrt{u}\left(1-\gamma_{k}\right)\left\|d_{0}\right\|+u\left\|w_{\gamma_{k}}\right\|+\frac{u}{k}, \quad \forall u \in\left[u_{k+1}, u_{k}\right)
$$

from which we get $x_{u}=x_{0}+\sqrt{u} d_{0}+o(\sqrt{u})$. Also, a second order expansion implies that for $u \in\left[u_{k+1}, u_{k}\right)$ we have

$$
f\left(x_{u}, u\right)=f\left(x_{0}, 0\right)+u\left[f^{\prime}\left(x_{0}, 0\right)\left(w_{\gamma_{k}}, 1\right)+\frac{1}{2} f_{x}^{\prime \prime}\left(x_{0}, 0\right) d_{0} d_{0}\right]+o(u)
$$

so that

$$
\begin{aligned}
f\left(x_{u}, u\right) & =f\left(x_{0}, 0\right)+u \Psi_{f}\left(w_{0}, d_{0}\right)+o(u) \\
& =v(0)+u v(\tilde{L})+o(u)=v(u)+o(u) .
\end{aligned}
$$

The conclusion follows.

\section{Perturbation analysis assuming nonexistence of multipliers.}

4.1. Preliminaries. In this section we analyze the situation when the set of multipliers $\Lambda_{0}$ is empty, extending the theory of perturbed singular nonlinear programs of [3]. The qualitative behaviour is radically different from the case studied in $\S 3$, so that we are led to introduce some new objects. Indeed, if $\Lambda_{0}$ is empty we have $v(L)=-\infty$ and by part $I$ it follows that $v^{\prime}(0)=-\infty$.

We will check that, under suitable second order assumptions, the variation of the cost is of order $O(\sqrt{u})$. This leads us to define, analogously to the Dini derivatives, the following quantities:

$$
\begin{aligned}
& v^{\#}(0):=\limsup _{u \downarrow 0} \frac{v(u)-v(0)}{\sqrt{u}}, \\
& v_{\#}(0):=\liminf _{u \downarrow 0} \frac{v(u)-v(0)}{\sqrt{u}} .
\end{aligned}
$$

We define the singular Lagrangian, the set of singular multipliers (at $x_{0}$, for problem $\left(P_{0}\right)$ ) and the set of normalized singular multipliers as:

$$
\begin{aligned}
\hat{\mathcal{L}}(x, \lambda, u) & :=\langle\lambda, G(x, u)\rangle, \\
\Lambda^{s} & :=\left\{\lambda \in Y^{*} \backslash\{0\}: \lambda \in N_{K}\left(G\left(x_{0}, 0\right)\right), \hat{\mathcal{C}}_{x}^{\prime}\left(x_{0}, \lambda, 0\right)=0\right\}, \\
\Lambda_{N}^{s} & :=\left\{\lambda \in \Lambda^{s}:\|\lambda\| \leq 1\right\} .
\end{aligned}
$$

The next proposition shows that $\Lambda_{0}$ and $\Lambda^{s}$ are both empty only in some very special situations.

Proposition 4.1. If both $\Lambda_{0}$ and $\Lambda^{s}$ are empty, then the set

$$
\mathcal{A}:=\mathbb{R}_{+}\left[K-G\left(x_{0}, 0\right)\right]-G_{x}^{\prime}\left(x_{0}, 0\right) X
$$

is dense in $Y$ but not equal to $Y$.

Proof. If $\mathcal{A}=Y$ we know that $\Lambda_{0} \neq \phi[13,14]$. Suppose next that $\mathcal{A}$ is not dense in $Y$ and select $y \in Y$ not belonging to the closure of $\mathcal{A}$. By the Hahn-Banach theorem there exists $\lambda \in Y^{*} \backslash\{0\}$ such that

$$
\langle\lambda, y\rangle>\left\langle\lambda, t\left[k-G\left(x_{0}, 0\right)\right]-G_{x}^{\prime}\left(x_{0}, 0\right) w\right\rangle \text { for all } w \in X, k \in K, t>0 .
$$

Taking the supremum over $w \in X$, we get $\lambda \circ G_{x}^{\prime}\left(x_{0}, 0\right)=0$, and letting $t \uparrow \infty$ we deduce $\left\langle\lambda, k-G\left(x_{0}, 0\right)\right\rangle \leq 0$ for all $k \in I$ so that $\lambda \in N_{K}\left(G\left(x_{0}, 0\right)\right)$ and then $\Lambda^{3} \neq \phi$. o 
4.2. Upper estimate of the cost. In order to obtain upper estimates for $v^{\#}(0)$ we consider the following optimization problems:

$$
\min _{d \in C_{0}}\left\{f_{x}^{\prime}\left(x_{0}, 0\right) d: \frac{1}{2} G_{x}^{\prime \prime}\left(x_{0}, 0\right) d d \in T_{2}^{K}(d)-G^{\prime}\left(x_{0}, 0\right) X \times\{1\}\right\},
$$

and

$(\hat{D}) \quad \min _{d \in C_{0}}\left\{f_{x}^{\prime}\left(x_{0}, 0\right) d: \frac{1}{2} G_{x}^{\prime \prime}\left(x_{0}, 0\right) d d \in \overline{T_{2}^{K}(d)-G^{\prime}\left(x_{0}, 0\right) X \times\{1\}}\right\}$.

Problem $(\hat{L})$ gives in a natural way a primal upper-estimate of the value function (if we have in mind paths satisfying $(2.3)$ and $(2.4)$ ), whereas $(\hat{D})$ will provide a comparison with the estimate of $v_{\#}(0)$. We remark that, when $\Lambda^{s}$ is not empty, problem $(\hat{D})$ is equivalent to

$$
\left(\hat{D}^{\prime}\right) \min _{d \in C_{0}}\left\{f_{x}^{\prime}\left(x_{0}, 0\right) d: \hat{\mathcal{L}}_{u}^{\prime}\left(x_{0}, \lambda, 0\right)+\frac{1}{2} \hat{\mathcal{L}}_{x}^{\prime \prime}\left(x_{0}, \lambda, 0\right) d d \leq \sigma\left(\lambda, T_{2}^{K}(d)\right), \forall \lambda \in \Lambda^{s}\right\} .
$$

To prove this equivalence it suffices to check that the constraints in $(\hat{D})$ and $\left(\hat{D}^{\prime}\right)$ coincide, which follows from the next result applied with $y=G_{u}^{\prime}\left(x_{0}, 0\right)+\frac{1}{2} G_{x}^{\prime \prime}\left(x_{0}, 0\right) d d$.

Proposition 4.2. If $\Lambda^{s} \neq \phi$ then the following are equivalent

(a) $y \in \overline{T_{2}^{K}(d)-G_{x}^{\prime}\left(x_{0}, 0\right) X}$

(b) $(\lambda, y\rangle \leq \sigma\left(\lambda, T_{2}^{K}(d)\right)$ for all $\lambda \in \Lambda^{s}$.

Proof. Both $(a)$ and $(b)$ are false if $T_{2}^{K}(d)$ is empty so we may assume the contrary. The implication $(a) \Rightarrow(b)$ is straightforward and the converse follows by a separation argument: indeed, if $(a)$ fails we may find a strictly separating hyperplane, that is, $\lambda \in Y^{*} \backslash\{0\}$ and $\alpha \in \mathbb{R}$ such that

$$
\langle\lambda, y\rangle>\alpha \geq\left\langle\lambda, k-G_{x}^{\prime}\left(x_{0}, 0\right) w\right\rangle
$$

for all $k \in T_{2}^{K}(d), w \in X$. Taking supremum over $w \in X$ it follows that $\lambda \circ G_{x}^{\prime}\left(x_{0}, 0\right)=$ 0 and then taking supremum over $k$ we deduce

$$
\langle\lambda, y\rangle>\alpha \geq \sigma\left(\lambda, T_{2}^{K}(d)\right) .
$$

Using this and (P4) we get $\lambda \in N_{K}\left(G\left(x_{0}, 0\right)\right)$ so that $\lambda \in \Lambda^{s}$ and (4.16) contradicts (b). प

We now state the upper-estimate.

THEOREM 4.3. If (SDCQ) holds then

$$
v^{\#}(0) \leq v(\hat{L})=v(\hat{D}) \leq 0,
$$

so that when $v(\hat{L})$ is finite we have

$$
v(u) \leq v(0)+\sqrt{u} v(\hat{L})+o(\sqrt{u}) .
$$

In addition, $v(\hat{L})<0$ iff there exists a direction $d$ such that $f_{x}^{\prime}\left(x_{0}, 0\right) d<0$ and $T_{2}^{K}(d) \neq \phi$ 
Proof. We begin by showing $v^{\#}(0) \leq v(\hat{L}) \leq 0$. Let $d \in F(\hat{L})$ and select $w \in X$ such that $G^{\prime}\left(x_{0}, 0\right)(w, 1)+\frac{1}{2} G_{x}^{\prime \prime}\left(x_{0}, 0\right) d \bar{d} \in T_{2}^{K}(d)$. Using the restorability property we may find feasible paths of the form $x_{u}^{\gamma}=x_{0}+\gamma \sqrt{u} d+u w_{\gamma}+o(u)$ with $w_{\gamma} \rightarrow w$ as $\gamma \uparrow 1$. Expanding $f$ it follows that

$$
v(u) \leq f\left(x_{u}^{\gamma}, u\right)=f\left(x_{0}, 0\right)+\gamma \sqrt{u} f_{x}^{\prime}\left(x_{0}, 0\right) d+o(\sqrt{u})
$$

from which we deduce

$$
v^{\#}(0) \leq \gamma f_{x}^{\prime}\left(x_{0}, 0\right) d .
$$

Letting $\gamma \uparrow 1$ and then taking the infimum over $d \in F(\hat{L})$ we get $v^{\#}(0) \leq v(\hat{L})$. Moreover, $(P 2)$ implies $0 \in F(\hat{L})$ so that $v(\hat{L}) \leq 0$.

We prove next $v(\hat{L})=v(\hat{D})$. Since clearly $v(\hat{D}) \leq v(\hat{L})$ it suffices to show that $v(\hat{L}) \leq f_{x}^{\prime}\left(x_{0}, 0\right) d$ for each $d \in F(\hat{D})$. Let $d \in \bar{F}(\hat{D})$ and select sequences $k_{n} \in T_{2}^{K}(d), w_{n} \in X$ such that $\frac{1}{2} G_{x}^{\prime \prime}\left(x_{0}, 0\right) d d=\lim _{n}\left[k_{n}-G^{\prime}\left(x_{0}, 0\right)\left(w_{n}, 1\right)\right]$. Using $(P 2)$ we find that given any $t>0$ we will have for all $n$ large enough

$$
\frac{1}{2} t G_{x}^{\prime \prime}\left(x_{0}, 0\right) d d-t k_{n}+t G^{\prime}\left(x_{0}, 0\right)\left(w_{n}, 1\right) \in T_{K}\left(G\left(x_{0}, 0\right)\right)-G^{\prime}\left(x_{0}, 0\right) X \times\{1\}
$$

which rearranged gives

$$
\frac{1}{2} \frac{t}{1+t} G_{x}^{\prime \prime}\left(x_{0}, 0\right) d d \in \frac{t}{1+t} k_{n}+T_{K}\left(G\left(x_{0}, 0\right)\right)-G^{\prime}\left(x_{0}, 0\right) X \times\{1\} .
$$

Letting $d_{t}:=\sqrt{t /(1+t)} d$ and using $(P 1)$ and $(P 3)$ we deduce

$$
\frac{1}{2} G_{x}^{\prime \prime}\left(x_{0}, 0\right) d_{t} d_{t} \in T_{2}^{K}\left(d_{t}\right)-G^{\prime}\left(x_{0}, 0\right) X \times\{1\} .
$$

Hence $d_{t} \in F(\hat{L})$ and then

$$
v(\hat{L}) \leq f_{x}^{\prime}\left(x_{0}, 0\right) d_{t} .
$$

Letting $t$ tend to $+\infty$ we conclude $v(\hat{L}) \leq f_{x}^{\prime}\left(x_{0}, 0\right) d$ as required.

We conclude by proving the sufficient condition for $v(\hat{L})<0$ (the necessity is evident). If $d \in X$ is such that $f_{x}^{\prime}\left(x_{0}, 0\right) d<0$ and $T_{2}^{K}(d) \neq \phi$, from Lemma 3.4 we get $\alpha d \in F(\hat{L})$ for all $\alpha>0$ sufficiently small, so that $v(\hat{L}) \leq \alpha f_{x}^{\prime}\left(x_{0}, 0\right) d<0$.

REMARK. From the estimate (1.1) we already know that $v_{\#}(0) \leq 0$. Henceforth Theorem 4.3 improves the upper-estimate of the cost only if $v(\hat{L})<0$.

4.3. Lower estimates and expansion of solutions. As in the case when $\Lambda_{0} \neq \phi$, we will give a lower estimate of the cost which is sharp when the contribution of the curvature of $K$ happens to be null.

We consider the singular second order conditions

$$
(S S O C) \quad \text { there exist } \alpha, \epsilon>0 \text { s.t. } \sup _{\lambda \in \Lambda_{N}^{e}} \hat{\mathcal{L}}_{x}^{\prime \prime}\left(x_{0}, \lambda, 0\right) d d \geq \alpha\|d\|^{2} \quad \forall d \in C_{\epsilon} .
$$

Proposition 4.4. If (SSOC) holds, then for each $O(\sqrt{u})$-optimal path $x_{u}$ we have $x_{u}=x_{0}+O(\sqrt{u})$. 
Proof. Let $x_{u}$ be an $O(\sqrt{u})$-optimal path and let $\beta_{u}:=\left\|x_{u}-x_{0}\right\|, d_{u}:=\left(x_{u}-\right.$ $\left.x_{0}\right) / \beta_{u}$. For each $\lambda \in \Lambda_{N}^{*}$ we have

$$
\begin{aligned}
0 & \geq \hat{\mathcal{L}}\left(x_{u}, \lambda, u\right)-\hat{\mathcal{L}}\left(x_{0}, \lambda, 0\right), \\
& =u \hat{\mathcal{L}}_{u}^{\prime}\left(x_{0}, \lambda, 0\right)+\frac{\beta_{u}^{2}}{2} \hat{\mathcal{L}}_{x}^{\prime \prime}\left(x_{0}, \lambda, 0\right) d_{u} d_{u}+o(u)+o\left(\beta_{u}^{2}\right) .
\end{aligned}
$$

The small terms $o(u)$ and $o\left(\beta_{u}^{2}\right)$ may be chosen independent of $\lambda \in \Lambda_{N}^{s}$, so we may take supremum to deduce

$$
\beta_{u}^{2} \max _{\lambda \in \Lambda_{N}^{\prime}} \mathcal{L}_{x}^{\prime \prime}\left(x_{0}, \lambda, 0\right) d_{u} d_{u} \leq O(u)+o\left(\beta_{u}^{2}\right)
$$

If for some sequence $u_{n} \downarrow 0$ one has $\beta_{u_{n}}^{2} / u_{n} \mid \infty$, then for $n$ large enough $d_{u_{n}}$ is in $C_{c}$. With $(S S O C)$ and (4.18), we obtain a contradiction. $\square$

To obtain the desired lower estimate for $v_{\#}(0)$ we consider a relazed version of problem $(\hat{D})$, namely

$$
\min _{d \in C_{0}}\left\{f_{x}^{\prime}\left(x_{0}, 0\right) d: \frac{1}{2} G_{x}^{\prime \prime}\left(x_{0}, 0\right) d d \in \overline{T_{K}\left(G\left(x_{0}, 0\right)\right)-G^{\prime}\left(x_{0}, 0\right) X \times\{1\}}\right\} .
$$

As for problem $(\hat{D})$, when $\Lambda^{s}$ is not empty one may use Proposition 4.2 (with $d=0$ ) to derive the following equivalent formulation for $(\hat{R})$ :

$$
\min _{d \in C_{0}}\left\{f_{x}^{\prime}\left(x_{0}, 0\right) d: \hat{\mathcal{L}}_{u}^{\prime}\left(x_{0}, \lambda, 0\right)+\frac{1}{2} \hat{\mathcal{L}}_{x}^{\prime \prime}\left(x_{0}, \lambda, 0\right) d d \leq 0 \text { for all } \lambda \in \Lambda^{s}\right\} .
$$

Comparing with $\left(\hat{D}^{\prime}\right)$ and using $(\mathrm{P} 4)$, we see that $F\left(\hat{D}^{\prime}\right) \subset F\left(\hat{R}^{\prime}\right)$. As these two problems have the same cost, it follows that

$$
v(\hat{R})=v\left(\hat{R}^{\prime}\right) \leq v\left(\hat{D}^{\prime}\right)=v(\hat{D}) .
$$

Proposition 4.5. Assume there exists an o $(\sqrt{u})$-optimal path $x_{u}$. If $(S S O C)$ is satisfied then $v_{\#}(0)>-\infty$. Moreover, if any of the two following properties hold

(a) the path may be expanded as $x_{u}=x_{0}+\sqrt{u} d_{0}+o(\sqrt{u})$,

(b) $X$ is reflexive and for each $\lambda \in \Lambda^{s}$ the mapping $d \rightarrow \hat{\mathcal{L}}_{x}^{\prime \prime}\left(x_{0}, \lambda, 0\right) d d$ is weakly then

l.s.c. at every $d_{0} \in C_{0}$,

$$
v_{\#}(0) \geq v(\hat{R}) .
$$

Proof. By Proposition 4.4 we have $x_{u}=x_{0}+O(\sqrt{u})$ and then

$$
v(u)=f\left(x_{u}, u\right)+O(\sqrt{u})=f\left(x_{0}, 0\right)+O(\sqrt{u})
$$

so that $v_{\#}(0)>-\infty$.

Now let us choose $u_{n} \downarrow 0$ realizing the lower limit $v_{\#}(0)$ and let $d_{n}:=\left(x_{u_{n}}-\right.$ $\left.x_{0}\right) / \sqrt{u_{n}}$. When $(a)$ holds we have $d_{n} \rightarrow d_{0}$, while in case $(b)$ we may assume that $d_{n}-d_{0}$ for some $d_{0} \in X$. In both cases, $d_{0} \in C_{0}$ and we have

$$
v_{\#}(0)=f_{x}^{\prime}\left(x_{0}, 0\right) d_{0} .
$$


On the other hand for all $\lambda \in \Lambda^{s}$

$$
\begin{aligned}
0 & \geq \hat{\mathcal{L}}\left(x_{u}, \lambda, u\right)-\hat{\mathcal{L}}\left(x_{0}, \lambda, 0\right) \\
& =u \hat{\mathcal{L}}_{u}^{\prime}\left(x_{0}, \lambda, 0\right)+\frac{u}{2} \hat{\mathcal{L}}_{x}^{\prime \prime}\left(x_{0}, \lambda, 0\right) d_{u} d_{u}+o(u)
\end{aligned}
$$

so that, using in case (b) the l.s.c. of $\hat{\mathcal{L}}_{x}^{\prime \prime}\left(x_{0}, \lambda, 0\right) d d$ we get

$$
0 \geq \hat{\mathcal{L}}_{u}^{\prime}\left(x_{0}, \lambda, 0\right)+\frac{1}{2} \hat{\mathcal{L}}_{x}^{\prime \prime}\left(x_{0}, \lambda, 0\right) d_{0} d_{0}
$$

It follows that $d_{0} \in F\left(\hat{R}^{\prime}\right)$. Combining with (4.19) we get

$$
v(\hat{R})=v\left(\hat{R}^{\prime}\right) \leq f_{x}^{\prime}\left(x_{0}, 0\right) d_{0}=v_{\#}(0)
$$

as was to be proved.

REMARK. Let us put together the different relations between optimal values. If $(S D C Q)$ holds, and the conclusion of Proposition 4.5 is true, then

$$
v(\hat{R})=v\left(\hat{R}^{\prime}\right) \leq v_{\#}(0) \leq v^{\#}(0) \leq v\left(\hat{D}^{\prime}\right)=v(\hat{D})=v(\hat{L}) \leq 0 .
$$

In our next statement, we give a condition under which the above optimal values are equal. This gives the first term of the expansion of the optimal value $v(u)$.

THEOREM 4.6. Assume the existence of an $O(\sqrt{u})$-optimal path $x_{u}$, (SSOC), $X$ reflexive, the l.s.c. of $d \rightarrow \hat{\mathcal{L}}_{x}^{\prime \prime}\left(x_{0}, \lambda, 0\right) d d$ for each $\lambda \in \Lambda^{s},(S D C Q)$ and finally

$$
0 \in T_{2}^{K}(d), \forall d \in C_{0} .
$$

Then $v(\hat{R})=v(\hat{D}), S(\hat{R})=S(\hat{D})$ and

$$
v(u)=v(0)+\sqrt{u} v(\hat{D})+o(\sqrt{u}) .
$$

Proof. The equivalence between $(\hat{R})$ and $(\hat{D})$ follows by noticing that when $0 \in$ $T_{2}^{K}(d)$ then (see [8, Prop. 3.1])

$$
T_{2}^{K}(d)=\overline{T_{K^{K}}\left(G\left(x_{0}, 0\right)\right)-\mathrm{IR}_{+} G_{x}^{\prime}\left(x_{0}, 0\right) d}
$$

from which we deduce

$$
\overline{T_{2}^{K}(d)-G^{\prime}\left(x_{0}, 0\right) X \times\{1\}}=\overline{T_{K}\left(G\left(x_{0}, 0\right)\right)-G^{\prime}\left(x_{0}, 0\right) X \times\{1\}} .
$$

The expansion of $v(u)$ then follows from Theorem 4.3 and Proposition 4.5.

5. Appendix: Checking the strong directional constraint qualification. We give some sufficient conditions allowing to check $(S D C Q)$ in the case of decomposed constraints of the form: $Y:=Y_{1} \times Y_{2}$ with $Y_{1}$ and $Y_{2}$ Banach spaces, $K:=K_{1} \times K_{2}$ with $K_{1}$ and $K_{2}$ closed convex subsets of $Y_{1}$ and $Y_{2}$. We denote $G=\left(G_{1}, G_{2}\right)$ the components of $G$ and we consider the decomposed directional constraint qualification: $(D D C Q) \quad \begin{cases}(\text { i }) & 0 \in \operatorname{int}\left[G_{1}\left(x_{0}, 0\right)+G_{1}^{\prime}\left(x_{0}, 0\right) X \times\{0\}-K_{1}\right], \\ \text { (ii) } & \text { There exists } \bar{w} \in X \text { such that } G_{1}^{\prime}\left(x_{0}, 0\right)(\bar{w}, 1) \in \operatorname{Rec}\left(K_{1}\right) \text { and } \\ & G_{2}\left(x_{0}, 0\right)+\alpha G_{2}^{\prime}\left(x_{0}, 0\right)(\bar{w}, 1) \in \operatorname{int} K_{2} \text { for some } \alpha>0 .\end{cases}$ 
where $\operatorname{Rec}\left(K_{1}\right)$ denotes the recession cone of $K_{1}$, that is

$$
\operatorname{Rec}\left(K_{1}\right):=\limsup _{t \rightarrow \infty} \frac{K_{1}}{t} .
$$

In order to illustrate this condition, let us mention two particular cases. The first one is when $K_{2}=Y_{2}$ so that the constraint is only with $K_{1}$. Then $(D D C Q)$ reduces to Robinson's condition [14]. The second case is when $K_{1}=\{0\}$. Then $(D D C Q)$ (i) amounts to the surjectivity of $G_{1 x}^{\prime}\left(x_{0}, 0\right)$, and $(D D C Q)$ appears as a natural generalization of Gollan's condition [10] used in the afore mentioned literature devoted to nonlinear programming.

THEOREM 5.1. (DDCQ) implies (SDCQ).

Proof. We first prove that $x_{0}$ is restorable. Let $x_{u}$ be a path satisfying (2.3) and (2.4). Choose $w_{\gamma}:=\gamma^{2} w+\left(1-\gamma^{2}\right) \bar{w}$ and consider

$$
y_{u}:=x_{0}+\gamma \sqrt{u} d+u w_{\gamma} .
$$

Expanding in scries we get

$$
\begin{aligned}
G\left(y_{u}, u\right)= & G\left(x_{0}, 0\right)+\gamma \sqrt{u} G_{x}^{\prime}\left(x_{0}, 0\right) d+u \Psi_{G}\left(w_{\gamma}, \gamma d\right)+o(u), \\
= & G\left(x_{0}, 0\right)+\gamma \sqrt{u} G_{x}^{\prime}\left(x_{0}, 0\right) d+\gamma^{2} u \Psi_{G}(w, d)+ \\
& +\left(1-\gamma^{2}\right) u G^{\prime}\left(x_{0}, 0\right)(\bar{w}, 1)+o(u), \\
= & G\left(x\left(\gamma^{2} u\right), \gamma^{2} u\right)+\left(1-\gamma^{2}\right) u G^{\prime}\left(x_{0}, 0\right)(\bar{w}, 1)+o(u) .
\end{aligned}
$$

Using $(D D C Q)(i i)$ and $(2.4)$ we deduce $d\left(G_{1}\left(y_{u}, u\right), K_{1}\right)=o(u)$. Then $(D D C Q)(i)$ allows us to use Robinson's theorem to find a small correction $x_{u}^{\gamma}$ of $y_{u}$,

$$
x_{u}^{\gamma}=x_{0}+\gamma \sqrt{u} d+u w_{\gamma}+o(u),
$$

such that $G_{1}\left(x_{u}^{\gamma}, u\right) \in K_{1}$.

Expanding $G_{2}\left(x_{u}^{\gamma}, u\right)$ as above, we get

$$
G_{2}\left(x_{u}^{\gamma}, u\right)=G_{2}\left(x\left(\gamma^{2} u\right), \gamma^{2} u\right)+\left(1-\gamma^{2}\right) u G_{2}^{\prime}\left(x_{0}, 0\right)(\bar{w}, 1)+o(u)
$$

so that letting $z:=G_{2}^{\prime}\left(x_{0}, 0\right)(\bar{w}, 1)$ and using (2.4) we have

$$
G_{2}\left(x_{t}^{\gamma}, u\right)=t_{u}+\left(1-\gamma^{2}\right) u z+o(u)
$$

for some $t_{u} \in K_{2}, t_{u} \rightarrow G_{2}\left(x_{0}, 0\right)$. Moreover, letting $\alpha_{u}:=\left(1-\gamma^{2}\right) u / \alpha$ we may write $G_{2}\left(x_{u}^{\gamma}, u\right)=\left(1-\alpha_{u}\right) t_{u}+\alpha_{u} r_{u}$ with

$$
r_{u}=t_{u}+\alpha z+\alpha o(u) /\left(1-\gamma^{2}\right) u=t_{u}+\alpha z+o(1) .
$$

By $(D D C Q)(i)$ we have $r_{u} \in K_{2}$ for $u$ small, and since also $t_{u} \in K_{2}$ and $\alpha_{u} \in(0,1)$, it follows that $G_{2}\left(x_{u}^{\gamma}, u\right) \in K_{2}$. Hence $x_{u}^{\gamma}$ is a feasible path and $x_{0}$ is restorable.

We now check that $(D C Q)$ is satisfied. By $(D D C Q)(i)$ (see [14]) there exist $\epsilon>0$ and $\beta>0$ such that, whenever $y_{1} \in Y_{1}$ satisfies $\left\|y_{1}\right\|<\epsilon$, there exists $\hat{d} \in X$ and $k_{1} \in K_{1}$ such that $\|\hat{d}\|<\beta\left\|y_{1}\right\|$ and

$$
G_{1}\left(x_{0}, 0\right)+G_{1}^{\prime}\left(x_{0}, 0\right)(\hat{d}, 0)-k_{1}=y_{1} .
$$


Now take $d$ of the form $d=\hat{d}+\alpha \bar{w}$. then

$$
G_{1}\left(x_{0}, 0\right)+G_{1}^{\prime}\left(x_{0}, 0\right)(d, \alpha)-\left[k_{1}+\alpha G^{\prime}\left(x_{0}, 0\right)(\bar{w}, 1)\right]=y_{1}
$$

and

$G_{2}\left(x_{0}, 0\right)+G_{2}^{\prime}\left(x_{0}, 0\right)(d, \alpha)-y_{2}=G_{2}\left(x_{0}, 0\right)+\alpha G_{2}^{\prime}\left(x_{0}, 0\right)(\bar{w}, 1)+G_{2}^{\prime}\left(x_{0}, 0\right)(\hat{d}, 0)-y_{2}$.

We may choose $\epsilon$ small so that for all $\left\|y_{1}\right\|<\epsilon,\left\|y_{2}\right\|<\epsilon$ we have $\left\|G_{2}^{\prime}\left(x_{0}, 0\right)(\hat{d}, 0)-y_{2}\right\|$ small enough to deduce, using $(D D C Q)(i i)$, that the left hand-side above is in $K_{2}$. From this $(D C Q)$ follows easily. $\square$

We note that we do not know (even for nonlinear programming problems) if the property $d\left(G\left(x_{0}+\sqrt{u} d+u w, u\right), K\right)=o(u)$ together with $(D C Q)$ suffices or not to construct a feasible path of the form $x_{u}=x_{0}+\sqrt{u} d_{0}+u w+o(u)$ (without $\gamma$ and $\left.w_{\gamma}\right)$.

Proposition 5.2. If $K:=\{0\} \times K_{2}$ with int $\left(K_{2}\right)$ nonempty, then $(D C Q)$, $(S D C Q),(D D C Q)$ are equivalent and are satisfied iff the condition below $(E D C Q)$ holds:

$(E D C Q) \quad \begin{cases}(i) & G_{1}^{\prime}\left(x_{0}, 0\right) X \times\{0\}=Y_{1}, \\ (\text { ii }) & \text { There exists } \bar{w} \in \text { such that } G_{1}^{\prime}\left(x_{0}, 0\right)(\bar{w}, 1)=0 \text { and } \\ & G_{2}\left(x_{0}, 0\right)+\alpha G_{2}^{\prime}\left(x_{0}, 0\right)(\bar{w}, 1) \in \operatorname{int} K_{2} \text { for some } \alpha>0 .\end{cases}$

Proof. Obviously each of the conditions $(D C Q),(S D C Q),(D D C Q),(E D C Q)$ is a consequence of the one that follows. Therefore it suffices to prove that $(D C Q)$ implies $(E D C Q)$. From $(D C Q), G_{1}^{\prime}\left(x_{0}, 0\right) X \times(0, \infty)$ contains a neighborhood of 0 . Being a cone, this set is equal to $Y_{1}$. In particular there exist $d_{0} \in X, \alpha_{0}>0$ such that $G_{1}^{\prime}\left(x_{0}, 0\right)\left(d_{0}, \alpha_{0}\right)=0$, i.e. $G_{u}^{\prime}\left(x_{0}, 0\right) \in G_{1}^{\prime}\left(x_{0}, 0\right) X \times\{0\}$. We deduce

$$
Y_{1}=G_{1}^{\prime}\left(x_{0}, 0\right) \lambda \times(0, \infty)=G_{1}^{\prime}\left(x_{0}, 0\right) X \times\{0\},
$$

i.e. $(E D C Q)(i)$ holds. Now pick $a \in \operatorname{int}\left(K_{2}\right)$, close enough to $G_{2}\left(x_{0}, 0\right)$ so that there exist $d \in X$ and $\tilde{\alpha}>0$ such that $\left(0, a-G_{2}\left(x_{0}, 0\right)\right) \in G\left(x_{0}, 0\right)+G^{\prime}\left(x_{0}, 0\right)(d, \tilde{\alpha})-K$. It is easily checked that $(E D C Q)(i i)$ is satisfied with $\bar{w}:=d / \tilde{\alpha}, \alpha:=\tilde{\alpha} / 2$. $\square$

\section{REFERENCES}

[1] A. Auslender and R. Cominetti, First and second order sensitivity analysis of nonlinear programs under directional constraint qualification conditions, Optimization 21 (1990), pp. 351-363.

[2] L. Barbet, Etude de sensibilité differentielle dans un probleme d'optimisation paramétré avec contraintes en dimension infinie, Thesis, Université de Poitiers (1992).

[3] J.F. Bonnans, Directional derivatives of optimal solutions in smooth nonlinear programining, J. Optimiz. Theory Appl. 73(1) (1992), pp. 27-45.

[4] J.F. Bonnans and R. Cominetti, Perturbed optimization in Banach spaces $I$ : A general theory based on a weak directional constraint qualification, INRIA report 2024, (1993).

[5] J.F. Bonnans and R. Cominetti, Perturbed optimization in Benach speces III: semi-infinite programming, INRIA report, to appear.

[6] J.F. Bonnans, A.D. Ioffe and A. Shapiro, Expansion of exact and approximate solutions in nonlinear programming, in Proc. French-German Conference in Optimization, D. Pallaschke ed., Lecture Notes in Economics and Math. Systems, Springer-Verlag (1992).

[7] J.F. Bonnans and A. Slıapiro, Sensitivity analysis of paranetrized programs under cone constraints, SIAM J. Control \& Opt. 30(6) (1992), pp. 1409-1422.

[8] R. Cominetti, Metric regularity, tangent sets and second order optimality conditions, Applied Math. \& Opt. 21 (1990), pp. 265-287. 
[9] J. Gauvin and R. Janin, Directional behaviour of optimal solutions in nonlinear mathematical progranıming, Math. Oper. Res. 13(4) (1988), pp. 629-649.

[10] B. Gollan, On the marginal function in nonlinear programming, Math. Oper. Res. 9 (1984), pp. 208-221.

[11] A.D. Ioffe, Variational analysis of a composite function: a formula for the lower second order epi-derivative, J. Math. Anal. Appl. 160 (1990), pp. 379-405.

[12] A.D. Ioffe, Variational analysis of a composite function : perturbations, value function and sensitivity. Preprint, Haifa MT $\mathbf{8 8 0 .}$

[13] H. Maurer and J. Zowe, First and second order necessary and sufficient optimality conditions for infinite dimensional programming problems, Math. Prog., 16 (1979), pp. 98-110.

[14] S.M. Robinson, Stability theorems for systems of inequalities. Part II: differentiable nonlinear systems, SIAM J. Numer. Anal. 13(1976), pp. 497-513. 


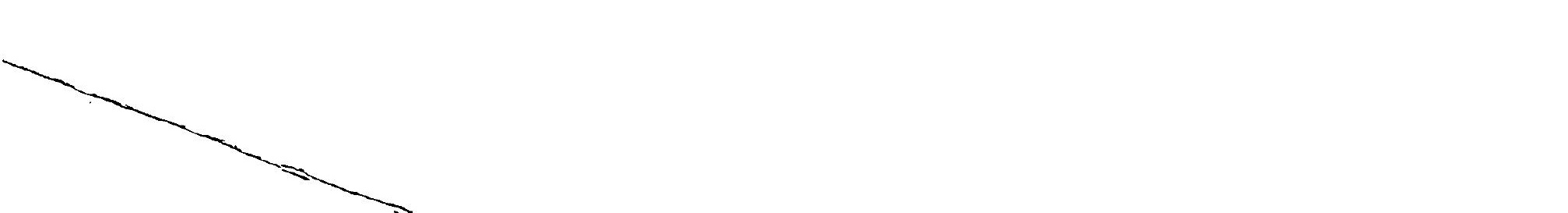




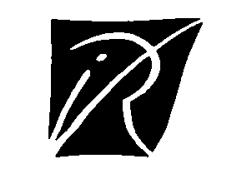

Unité de Recherche INRIA Rocquencourt

Domaine de Voluceau - Rocquencourt - B.P. 105 - 78153 LE CHESNAY Cedex (France)

Lnité de Recherche INRIA L.orraine Technopôle de Nancy-Brabois - Campus Scientifique

615. rue du Jardin Botanique - B.P. 101 - 54602 VII.L.LRS I.1:S NA.VCY Cedex (France)

Lnité de Recherche IVRIA Rennes IRISA. Campus Universitaire de Beaulieu 35042 RENNES Cedex (France)

Unité de Recherche INRIA Rhône-Alpes 46, avenue Félix Viallet - 38031 GRE.NOBLE (edex (France)

Linité de Recherche INRIA Sophia Antipolis 2004, route des Lucioles - B.P. 93 - 06902 SOPHIA ANTlIPOLIS Cedex (France)

\section{EDITEUR}

INRIA - Domaine de Voluceau - Rocquencourt - B.P. 105 - 78153 LE CHESNAY Cedex (France)

ISSN $0249-6399$ 